\title{
Assessing the umbrella value of a range-wide conservation network for jaguars (Panthera onca)
}

\author{
Daniel Thornton, ${ }^{1,6}$ Kathy Zeller,,${ }^{2,3}$ Carlo Rondinini, ${ }^{4}$ Luigi Boitani, ${ }^{4}$ Kevin Crooks, ${ }^{5}$ \\ Christopher Burdett, ${ }^{5}$ Alan Rabinowitz, ${ }^{2}$ and Howard Quigley ${ }^{2}$ \\ ${ }^{1}$ School of Environment, Washington State University, PO Box 642812, Pullman, Washington, USA \\ ${ }^{2}$ Panthera, 8 West 40th Street, New York, New York, USA \\ ${ }^{3}$ Department of Environmental Conservation, University of Massachusetts, 160 Holdsworth Way, Amherst, Massachusetts \\ 01003 USA \\ ${ }^{4}$ Department of Biology and Biotechnologies, Sapienza Università di Roma, Viale Università 32, Roma, 00185 Italy \\ ${ }^{5}$ Department of Biology, Colorado State University, Campus Delivery 1878, Fort Collins, Colorado, USA
}

\begin{abstract}
Umbrella species are employed as conservation short-cuts for the design of reserves or reserve networks. However, empirical data on the effectiveness of umbrellas is equivocal, which has prevented more widespread application of this conservation strategy. We perform a novel, large-scale evaluation of umbrella species by assessing the potential umbrella value of a jaguar (Panthera onca) conservation network (consisting of viable populations and corridors) that extends from Mexico to Argentina. Using species richness, habitat quality, and fragmentation indices of $\sim 1500$ co-occurring mammal species, we show that jaguar populations and corridors overlap a substantial amount and percentage of high-quality habitat for co-occurring mammals and that the jaguar network performs better than random networks in protecting high-quality, interior habitat. Significantly, the effectiveness of the jaguar network as an umbrella would not have been noticeable had we focused on species richness as our sole metric of umbrella utility. Substantial inter-order variability existed, indicating the need for complementary conservation strategies for certain groups of mammals. We offer several reasons for the positive result we document, including the large spatial scale of our analysis and our focus on multiple metrics of umbrella effectiveness. Taken together, our results demonstrate that a regional, single-species conservation strategy can serve as an effective umbrella for the larger community and should help conserve viable populations and connectivity for a suite of co-occurring mammals. Current and future range-wide planning exercises for other large predators may therefore have important umbrella benefits.
\end{abstract}

Key words: conservation network; habitat quality; jaguar; mammal; Panthera onca; surrogate; umbrella species.

\section{INTRODUCTION}

The use of surrogate species is a commonly applied strategy in conservation and management (Caro and O’Doherty 1999, Caro 2010). "Surrogate species" is a blanket term that encompasses several different concepts, including indicator species, umbrella species, keystone species, and flagship species, among others. Surrogates are used as short-cuts for monitoring biodiversity and ecosystem health, or for prioritizing areas for conservation, and thus are a key component of conservation efforts of a variety of organizations (Caro 2010). Despite their widespread usage, the effectiveness of the surrogate species strategy is contentious and faces theoretical (Saetersdal and Gjerde 2011) and empirical (Andelman and Fagan 2000, Bifolchi and Lodé 2005, Fleishman and Murphy 2009, Che-Castaldo and Neel

Manuscript received 1 April 2015; revised 28 August 2015; accepted 5 November 2015. Corresponding Editor: T. O'Brien.

${ }^{6}$ E-mail: daniel.thornton@wsu.edu
2012) challenges. This lack of certainty in the efficacy of surrogate species may contribute to the criticism of single species conservation efforts and increasing interest in more holistic ecosystem management approaches (Simberloff 1998, Mac Nally et al. 2002, Lindenmayer et al. 2007). Thus, there is a pressing need for more rigorous analyses of how surrogate species might be used to measure the success of conservation approaches and the circumstances under which such approaches will be most effective.

Umbrella species are intuitively appealing examples of a surrogate species. The umbrella species concept suggests that conservation planning based on the needs (e.g., habitat area and connectivity) of one species may benefit co-occurring species (Roberge and Angelstam 2004, Branton and Richardson 2010). Typically, umbrella species are organisms with large area requirements and/ or specialized habitat needs (Caro 2010; but see Fleishman et al. [2001] and Branton and Richardson [2010] for other possible characteristics of umbrella). Therefore, a 
protected area or reserve network that is designed to conserve viable populations of a particular umbrella species will simultaneously conserve viable populations of many other co-occurring species. However, echoing limitations of the surrogate species approach more generally, the utility of umbrella species as conservation tools remains poorly understood (Roberge and Angelstam 2004, Caro 2010). Results from studies examining umbrella species effectiveness are equivocal in terms of the utility of umbrellas as both tools for reserve selection (Andelman and Fagan 2000, Bifolchi and Lodé 2005, Branton and Richardson 2010, Sattler et al. 2013) and the maintenance of connectivity between reserves (Minor and Lookingbill 2010, Epps et al. 2011, Cushman and Landguth 2012, Brodie et al. 2014).

Two potential factors may contribute to variability in umbrella effectiveness. The first is spatial scale; most studies of umbrella effectiveness compare species richness in one or several reserves (or corridors) that contain an umbrella, with one or several that do not (Branton and Richardson 2010). However, umbrella species may be most useful as conservation tools at larger scales, for example, at regional scales or across an entire reserve network (Noss et al. 1996, Carroll et al. 2001, Rondinini and Boitani 2006). The second factor is the response variable used to determine the effectiveness of umbrellas. In many previous studies, the response that is measured is richness of co-occurring species (Branton and Richardson 2010, Caro 2010). However, other types of response may be more important, given that the goal of an umbrella is to maintain viable populations of cooccurring species. These other measures of umbrella efficacy include population size and habitat quality of co-occurring species (Branton and Richardson 2014), or degree of conservation network overlap (Rondinini and Boitani 2006). Different patterns in umbrella effectiveness may emerge depending on the type of response analyzed (Branton and Richardson 2014).

Large carnivores are often employed as umbrella species due to their substantial area needs and general sensitivity to human disturbance, but little is known about their actual umbrella value (Sergio et al. 2006, 2008). Several recent attempts at range-wide conservation planning for large carnivores have been conducted (Sanderson et al. 2002, IUCN 2006, Rabinowitz and Zeller 2010), which provide an opportunity to examine the umbrella-value of carnivores at large spatial extents. Moreover, recently developed global datasets on habitat suitability and fragmentation for mammals (Crooks et al. 2011, Rondinini et al. 2011) provide the ability to examine not only how carnivores function as umbrellas for conserving species richness of co-occurring mammals, but perhaps more importantly, how they serve as umbrellas for maintaining high-quality, core habitat for sympatric mammals.

The goal of our study was to examine the potential umbrella effectiveness of a conservation network designed to meet the needs of a single species, the jaguar (Panthera onca). This range-wide network (Fig. 1) extends from Mexico in the north to Argentina in the south and consists of viable jaguar populations (Jaguar Conservation Units; JCUs) and jaguar movement corridors that connect those populations. We ask the questions: (1) To what extent can the JCUs and jaguar

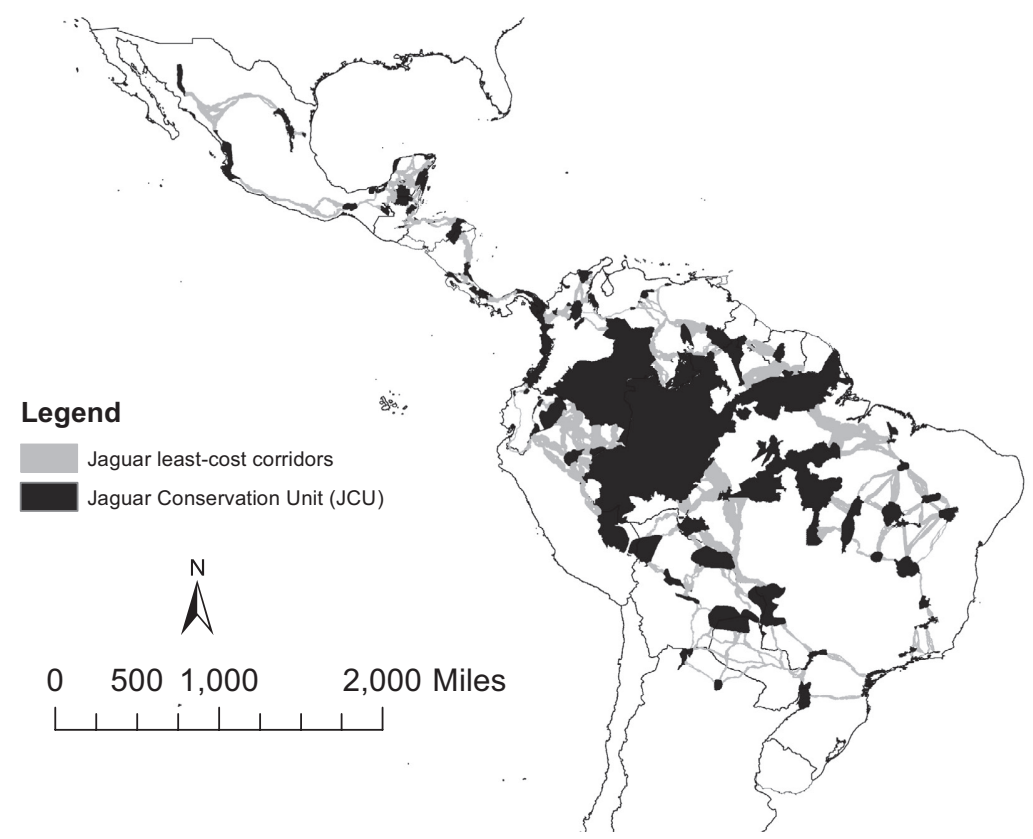

FIG. 1. A map of the jaguar conservation network for the Americas. The network consists of Jaguar Conservation Units (JCUs; in black), which maintain viable populations of jaguars, and jaguar corridors (in gray), linking the JCUs. 
corridors act to maintain species richness and highquality habitat for co-occurring Latin American mammals? (2) How well might jaguar corridors act to conserve connectivity (e.g., areas of low fragmentation) for co-occurring mammals? (3) How well can JCUs and corridors function as umbrellas when compared to a random selection of reserves or corridors?

\section{Materials And Methods}

\section{Modeling Jaguar Conservation Units and corridors}

The process of developing a range-wide conservation plan for the jaguar began in 1999 when the Wildlife Conservation Society (WCS) and the Universidad Naciónal Autonoma de México, Mexico City, Mexico, convened 35 jaguar experts to implement a rigorous planning exercise (Sanderson et al. 2002). The knowledge of these experts was pooled to determine the geographic distribution of jaguars, point observations of occurrence, and most importantly, core populations of jaguars. These core populations, termed Jaguar Conservation Units (JCUs), were defined as (1) areas with stable prey community that contained a population of at least 50 breeding jaguars or (2) areas with fewer than 50 breeding jaguars but with sufficient habitat and prey base such that jaguar populations could increase under favorable conditions (Sanderson et al. 2002). Only 28\% of JCU area overlaps with existing protected areas. This planning process was updated in 2006 and resulted in a range-wide map of 90 JCUs (Zeller 2007), which has been further modified to result in the most recent final map of JCUs (Fig. 1). We acknowledge that the model of JCUs we use in this paper may not be completely accurate in identifying the exact spatial location of all viable jaguar populations and that local-level refinements are and will continue to occur (e.g., De Angelo et al. 2013). However, the JCU network we employ does provide a consistent, expert-validated, range-wide model with which to evaluate the broad-scale umbrella value of jaguars, and provides a good template for what may occur with other range-wide planning exercises.

An important component missing from the initial range-wide plan, and its subsequent refinement, was an evaluation of potential jaguar movement, or dispersal, between JCUs and their likely routes of movement. Accordingly, Rabinowitz and Zeller (2010) predicted corridors between JCUs via least-cost path modeling (Adriaensen et al. 2003). In this approach, resistance values, indexing travel costs for a particular species, are assigned to different environments on a landscape (e.g., habitat types; Beier et al. 2008). Then, modeling algorithms are used to determine the path of least resistance between two populations; this pathway defines the corridor with the lowest cost of movement and highest probability of survival and can be used to focus conservation efforts for that species. Given a lack of empirical information on how jaguars disperse across landscapes, expert opinion was used to derive the resistance values for the creation of jaguar least-cost corridors. Resistance maps were created by having 15 jaguar experts assign resistance values to movement according to six factors: land cover type, percent tree and shrub cover, elevation, distance from roads, distance from settlements, and human population density (Rabinowitz and Zeller 2010). Resistance values were summed for each $1-\mathrm{km}^{2}$ pixel across the jaguar's range, and least-cost pathways between the JCUs were developed using Cost-Distance and Corridor functions in ArcGIS (Rabinowitz and Zeller 2010). The end result of this analysis was a map representing landscape resistance to jaguar movements and identification of leastcost corridors linking JCUs (Fig. 1; note this is slightly modified from Rabinowitz and Zeller 2010; see Materials and Methods: Overlap with co-occurring mammals).

\section{Overlap with co-occurring mammals}

To analyze how the JCUs and jaguar corridors can function as umbrellas for species richness and habitat quality of co-occurring mammals, we used ArcGIS to overlap the JCUs and corridors with habitat suitability models for $\sim 1500$ mammals inhabiting Latin America. We emphasize that our analysis does not compare how well jaguars perform as umbrellas to other potential umbrella species, but rather analyzes the umbrella value of a range-wide conservation strategy designed around the needs of a single species. The habitat suitability models were created by Rondinini and co-authors (Rondinini et al. 2011) for almost all terrestrial mammals globally. Briefly, for each mammal species, habitat suitability models (at 300-m resolution) were developed that indicated areas of high-, medium-, and low-quality habitat. Suitability was based on habitat preferences and elevational restrictions given in the IUCN Red List species descriptions, and utilized global land cover, elevation, and hydrologic maps. The suitability models were restricted to the known range of each species to avoid extrapolating species presence beyond their known limits. High-quality habitat corresponded to preferred habitat for the species, medium-quality habitat corresponded to habitat where the species could be found, but not persist in the absence of primary habitat, and low-quality (unsuitable) habitat corresponded to habitat where the species would rarely or never be found (see Rondinini et al. 2011 for details). We used ArcGIS to extract the areal extent of high-, medium-, and low-quality habitat contained within JCUs and jaguar corridors for each mammal species. We also determined the number of different species that had at least some amount of habitat (of any quality) that fell within JCUs and corridors. This latter index corresponds to the typical umbrella species analysis, where range maps of co-occurring mammals are used to determine the species richness contained within a conservation network (e.g., Carroll et al. 2001). We analyzed Mexico/Central America and South American separately due to faunal distinctiveness in the two regions, as well as structural 
differences in the jaguar network (Mexico/Central America having much smaller JCUs on average than South America). For South America, we excluded the large Amazonian JCU (the largest JCU in Fig. 1) from the analysis, as we did not feel it was appropriate to include such a massively large JCU $\left(2278098 \mathrm{~km}^{2}\right)$ in the umbrella analysis. This JCU would unduly bias our analysis toward finding a positive umbrella effect, and it represents too large of an area to be feasibly targeted as part of a protected area network.

Although gaining an understanding of the mammalian species richness and areal extent of habitat contained within the jaguar conservation network is useful in understanding its umbrella value, we also wanted to determine the proportion of the total available habitat for each species that was contained within the JCUs and corridors. We defined the total amount of habitat available for each co-occurring mammal using two scenarios, one more conservative and one less conservative for assessing the umbrella value of jaguars. For the more conservative method, we assumed that amount of habitat available to be conserved for each co-occurring mammal was equivalent to the total amount of identified habitat within all of Latin America (Mexico through Argentina) for that species. Any habitat for mammals that fell outside that range (e.g., habitat in the USA for certain wide-ranging species) was not included in the calculation of available habitat. In this way, we were assessing the umbrella value of jaguars for Latin American mammals more generally, even for mammals that have all or part of their habitat falling outside the current range of the jaguar, which does not extend throughout all of Latin America. For the less conservative method, we assumed that the amount of habitat available to be conserved for each co-occurring mammal was equivalent to the total amount of habitat within the outer boundaries of the jaguar's current range in Latin America (Appendix S1: Fig. S1). We excluded habitat (or species) that fell outside of this outer boundary. This second analysis therefore represents the umbrella value of jaguars for those mammals within the overall planning area for the jaguar reserve network. Note that even this second method is still fairly conservative, as we are including in the analysis co-occurring mammals that could never be contained within a jaguar network because they fall outside current jaguar habitat (e.g., high elevation species) but still fall within the outer boundaries of jaguar range. Once the total amount of habitat available for each co-occurring species was defined, we divided the amount of high-, medium-, and low-quality habitat for each species contained within JCUs and corridors by the total amount available. This calculation provided an estimate of the proportion of the total available high-, medium-, and low-quality habitat for each co-occurring mammal that was contained with JCUs and jaguar corridors.

For the jaguar corridors, we performed a second overlap analysis with fragmentation models for all $\sim 1500$ co-occurring mammals. For each species, the fragmentation models indicate the value of the GISfrag metric, which gives the average Euclidean distance of all cells within a high-quality habitat patch to the nearest edge of the patch (see Crooks et al. 2011 for details). High values of the GISfrag metric therefore indicate more interior habitat (or lower degree of fragmentation), whereas low values indicate less interior habitat (or higher degree of fragmentation; Crooks et al. 2011). We calculated the mean value of the GISfrag metric for each mammal species within jaguar corridors as a measure of how well jaguar corridors conserved interior, core habitat for co-occurring mammals.

\section{Comparison with random models}

We compared how well JCUs and jaguar corridors performed as potential umbrellas for co-occurring mammals with randomly selected protected area networks and randomly generated least-cost corridors. To generate a random protected area network to compare with the JCUs, we randomly selected protected areas within the study region from the World Database of Protected Areas (see Fig. 2 for an example). We excluded marine reserves and randomly selected protected areas until the total area within the reserve network was approximately the same as within the JCUs. Because only $\sim 28 \%$ of JCU area falls within the protected area network of the study region, this random selection of protected areas resulted in a spatially distinct network from the JCUs for comparison of umbrella value (i.e., there was only partial overlap of the JCUs with the random protected area networks). The JCUs themselves were not included in the random selection. We created 10 randomly generated networks for both Mexico/Central America and South America to compare with the JCUs. We then overlapped these randomly generated networks with the Rondinini et al. (2011) habitat suitability models as described previously for the JCUs. Because the random reserve networks and JCUs were not equivalent in area, to compare their efficiency in conserving high-, medium-, and low-quality habitat for co-occurring mammals and for species richness more generally, we divided the areal extent of overlapping habitat and the number of species protected by the total area of the network. This value (which we term the "protected area efficiency") indicates the proportion of the network that contains high-, medium-, and low-quality habitat for each co-occurring mammal, or the average number of species protected per unit area of the network. We summarized data by mammalian order and across all mammals, with each randomly generated network representing a separate sampling occasion. We then determined if the protected area efficiency value for JCUs was higher than the 95\% confidence interval for the randomly generated networks, which would indicate that the JCUs were better than random networks in protecting species richness and habitat quality of co-occurring mammals. 


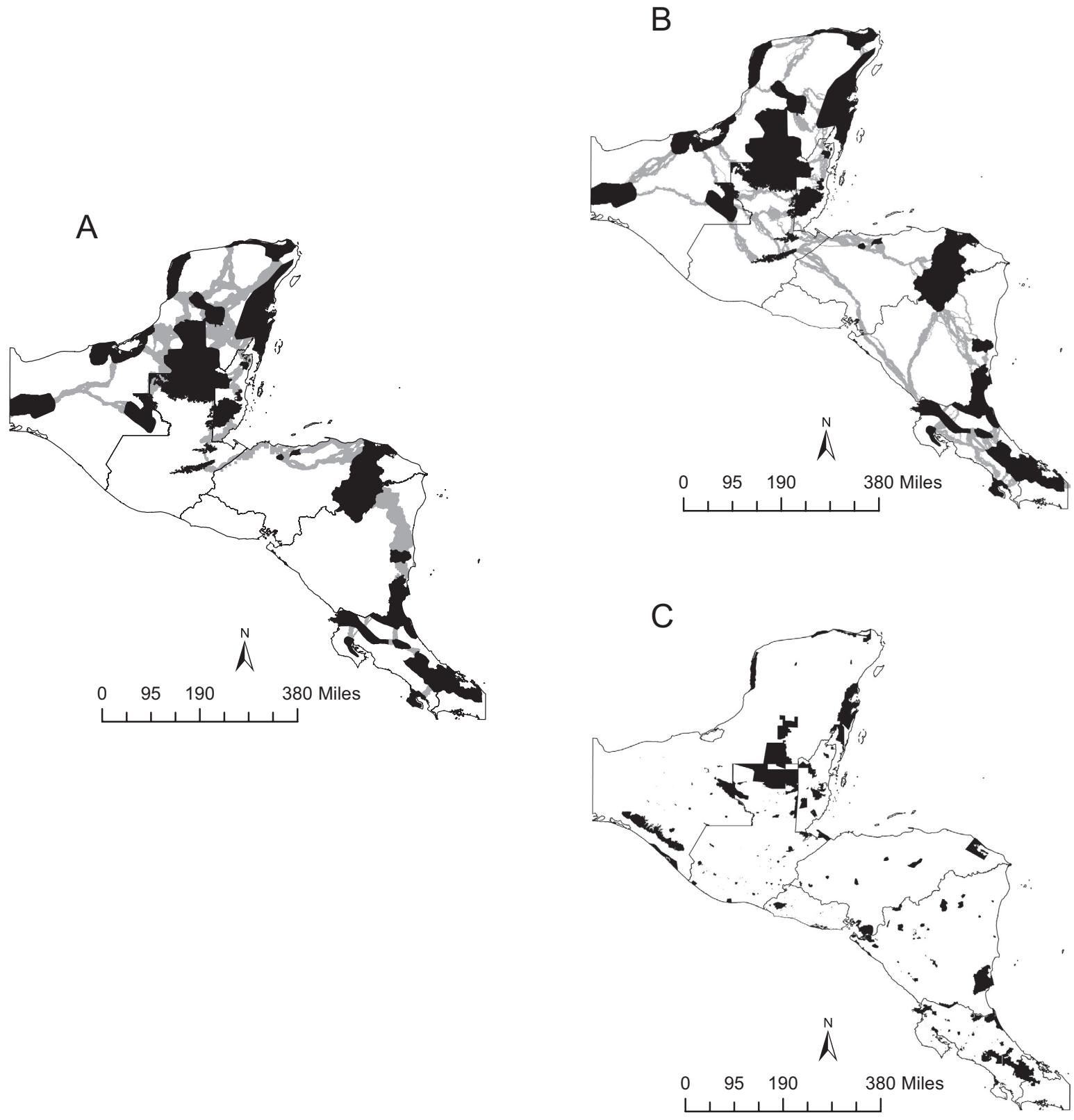

FIG. 2. Example comparison of jaguar network with random networks. Panel (A) is a map of actual JCUs (black) and modeled jaguar corridors (gray) for a section of Mexico/Central America, (B) a map of random generated corridors (gray) linking JCUs, and (C) a map of randomly selected protected areas (black) from the World Database of Protected Areas. Note that 10 corridor networks, and 10 protected area networks, were generated for comparison with the actual jaguar network.

To generate random least-cost corridor linkages between JCUs, we randomly permutated the jaguar costsurface used to create the jaguar corridor network. The cost-surface values were randomly sampled without replacement and the original cost-surface was reclassified to reflect the order of the randomly sampled cost values. Corridors were modeled between all JCUs across the random cost-surface using Cost-Distance and Corridor functions in ArcGIS. Note that for South American corridors, we used an earlier version of the jaguar corridor network than shown in Fig. 1. This earlier version was used to maintain consistency in the use of coarse-scaled environmental layers for generation of the real vs. random corridors.

We repeated the process of randomly generating corridors 10 times (see Fig. 2 for an example) for Mexico/ Central America and South America. Because the random corridor networks and jaguar corridors were not exactly equivalent in area, we repeated the process described for JCUs of dividing the amount of overlapping mammal 
habitat, as well as the number of species protected, by the total area of the network. This value (which we term the "corridor efficiency") indicates the proportion of the network that contains high-, medium-, and low-quality habitat for each co-occurring mammal or the average number of species protected per unit area of the network. We examined if the jaguar corridors had a better efficiency value for mammalian orders, and across all mammals, by comparing corridor efficiency values for jaguar corridors with $95 \%$ confidence intervals of the 10 randomly generated corridor networks.

\section{RESULTS}

\section{Overlap with co-occurring mammals}

We focus the results on how well the jaguar network overlapped high-quality habitat for co-existing mammals. Additional results on how the jaguar network protected low- and medium-quality habitat are in Appendix S2 (Fig. S1). Using our more conservative method to define available habitat, of the 583 mammal species with at least some high-quality habitat in all of Mexico/Central America, 423 (73\%) had some of that habitat contained within JCUs. Using our less conservative method to define available habitat, $81 \%$ of mammals with high-quality habitat available within the jaguar's range in Mexico/Central America have at least some of that habitat within JCUs. Jaguar corridors in Central America overlapped high-quality habitat for $45 \%$ and $50 \%$ of co-occurring mammal species, for more and less conservative estimates of availability, respectively. JCUs conserved a substantial proportion of the total available high-quality habitat for co-existing mammal species. The average proportion ( $\pm \mathrm{SD}$ ) of high-quality habitat that overlapped with JCUs was $0.22(0.27)$ and $0.25(0.26)$ across all species, for the more and less conservative estimates of available habitat, respectively. A substantial amount of variability in the proportion of high-quality habitat falling within JCUs occurred across mammalian orders, with average proportion ranging from 0.09 to 0.52 (Fig. 3; see Appendix S2: Fig. S2 for inter-order results based on the more conservative estimate of available habitat and Fig. S3 for inter-order results based on total area of the range in square kilometers contained within JCUs). In terms of total areal extent, JCUs conserved an average of $42018 \mathrm{~km}^{2}$ of high-quality habitat for co-occurring mammals in Mexico/Central America and jaguar corridors an additional $19327 \mathrm{~km}^{2}$.

Similar patterns are seen in South America. JCUs overlapped high-quality habitat for $70 \%$ and $81 \%$ of cooccurring mammal species for more and less conservative estimates of availability, respectively. In addition, jaguar corridors overlapping high-quality habitat for $67 \%$ and $75 \%$ of species for more or less conservative estimates, respectively. The average proportion of highquality habitat conserved within JCUs in South America was slightly lower than in Mexico/Central America, with averages of $0.15(0.19)$ and $0.18(0.20)$ across all species, for the more and less conservative definitions of available habitat. Again, substantial inter-order variability in the proportion of high-quality habitat overlapping JCUs was present, with average proportion ranging from 0 to 0.30 (Fig. 3; see Appendix S2: Fig. S2 for inter-order results based on the more conservative estimate of available habitat, and see Fig. S3 for inter-order results based on total area of the range in square kilometers contained within JCUs). In terms of total areal extent, JCUs conserved an average of $168198 \mathrm{~km}^{2}$ of high-quality habitat for co-occurring mammals in South America and jaguar corridors an additional $51107 \mathrm{~km}^{2}$.

\section{Comparison with random models}

JCUs in Mexico/Central America were less efficient than randomly generated protected area networks at overlapping absolute numbers of species. The number of species with at least some low-, medium-, or highquality habitat overlapping JCUs ( 1.69 species $\left./ 1000 \mathrm{~km}^{2}\right)$ fell below the $95 \%$ CI of the random networks $(2.74 \pm .05$ species $/ 1000 \mathrm{~km}^{2}$ ). However, JCUs were more efficient than random protected area networks at protecting highquality habitat for co-occurring mammals. JCU efficiency was greater than random networks across all species and for 10 of 11 mammalian orders (i.e., the efficiency value fell outside the $95 \%$ confidence interval of the efficiency value for the random networks; Fig. 4A). In contrast, JCU efficiency generally fell within, or just barely outside, the $95 \%$ CI of random networks for low- and medium-quality habitat (Appendix S2: Fig. S1). Similar overall patterns are seen for JCU efficiency in comparison to random networks for South America, with JCUs significantly less efficient in protecting species richness $\left(0.60\right.$ species $\left./ 1000 \mathrm{~km}^{2}\right)$ compared to random networks $\left(0.86 \pm 0.05\right.$ species $\left./ 1000 \mathrm{~km}^{2}\right)$, but more efficient in protecting high-quality habitat than random networks across all species. Moreover, JCUs in South America were more efficient at overlapping highquality habitat for 10 of 13 orders (Fig. 4B).

Jaguar corridors in Mexico/Central America were more efficient than randomly generated corridor networks at protecting a larger number of species within networks. The number of species with at least some low-, medium-, or high-quality habitat overlapping jaguar corridors $\left(3.78\right.$ species $/ 1000 \mathrm{~km}^{2}$ ) fell above the $95 \% \mathrm{CI}$ of the random networks $\left(3.5 \pm 0.05\right.$ species $\left./ 1000 \mathrm{~km}^{2}\right)$. Jaguar corridors were also more efficient than random corridor networks at protecting high-quality habitat across all species and for 10 of 11 mammalian orders (Fig. 4C). Moreover, jaguar corridors conserved more high-quality habitat within interior core (i.e., less fragmented) patches across all species, and for 10 of 11 orders, than random corridors (Fig. 5A). In South America, jaguar corridors were less efficient at protecting species richness (species richness within jaguar corridors, 0.35 species $/ 1000 \mathrm{~km}^{2}$; species richness random 


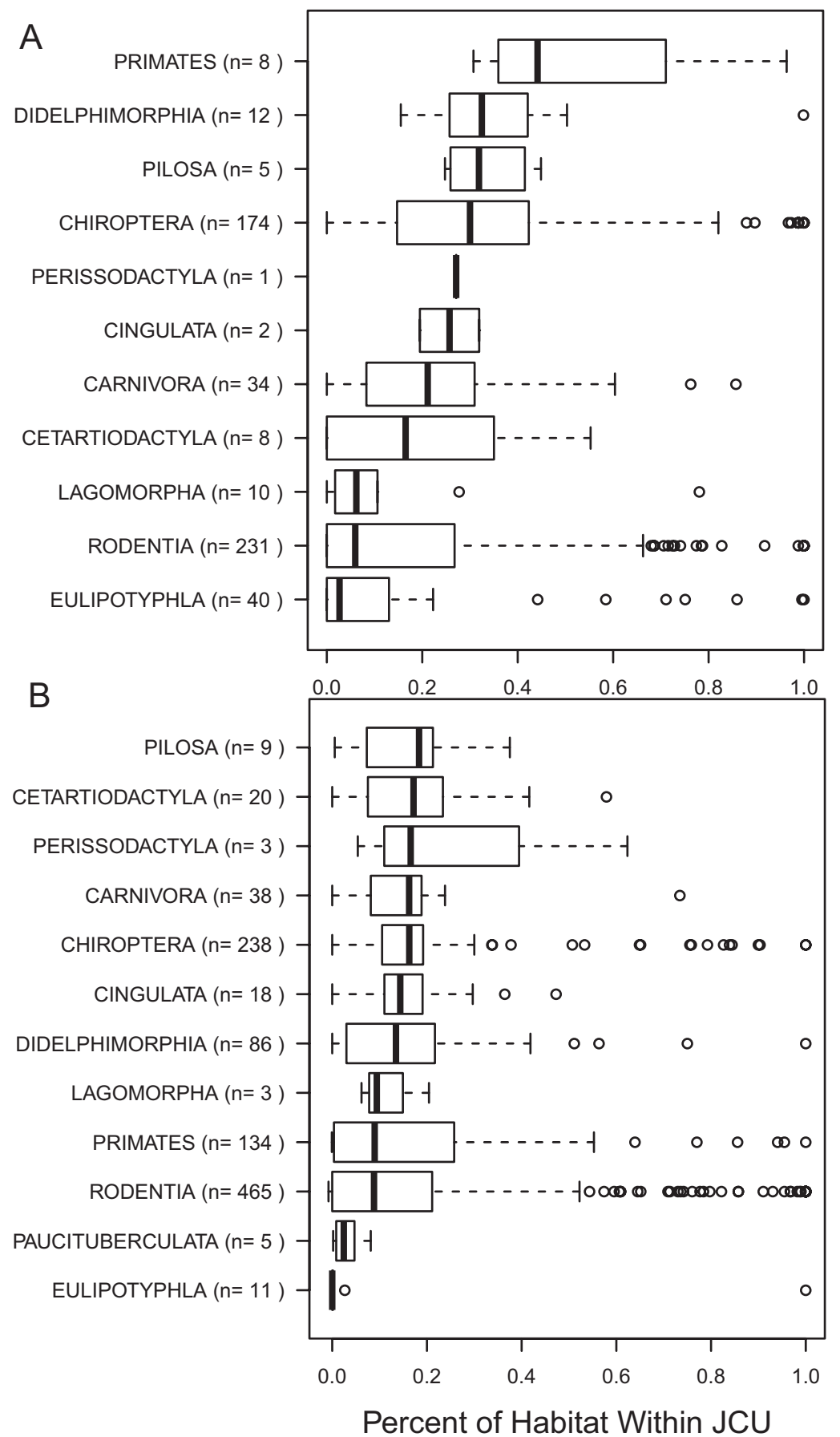

FIG. 3. Boxplots of the proportion of available high-quality habitat of co-occurring mammals contained within JCUs. Boxplots show the median (vertical black lines), upper and lower quartiles (open boxes), and variability outside those quartiles (whiskers). Panel (A) shows results from an analysis of Mexico/Central America, and (B) results from an analysis of South America. Available high-quality habitat was defined as the total amount of high-quality habitat of each species that fell within the outer boundary of the jaguar's range. Boxplots based on defining available high-quality habitat as all high-quality habitat within Latin America can be found in Appendix S2: Fig. S2.

corridors, $0.38 \pm 0.02$ species $/ 1000 \mathrm{~km}^{2}$ ). However, South American jaguar corridors were more efficient in protecting high-quality habitat than random corridors across all species and for 9 of 13 orders (Fig. 4D). South American jaguar corridors were also more efficient in protecting intact high-quality core habitat (Fig. 5B). 
A

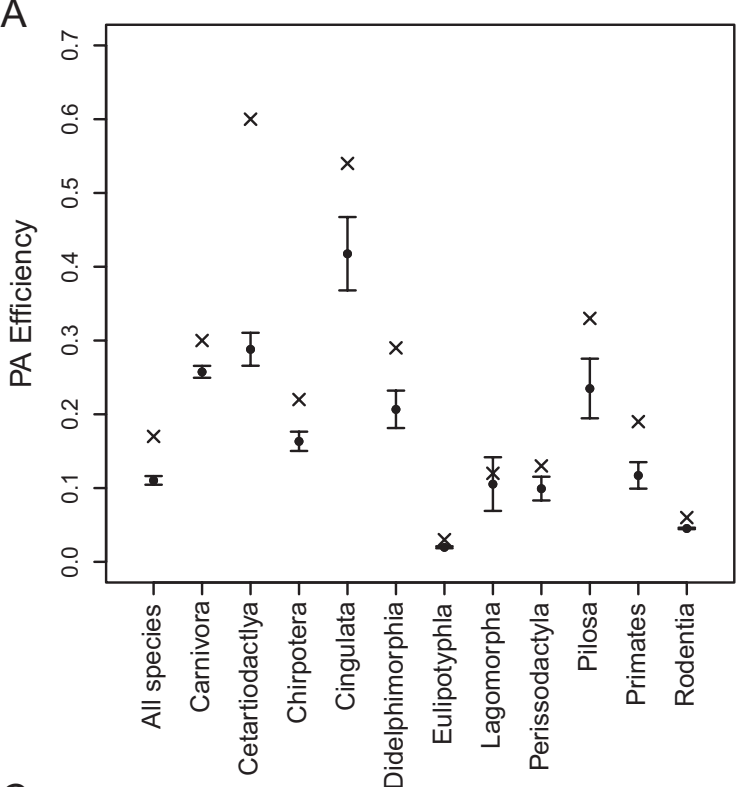

C

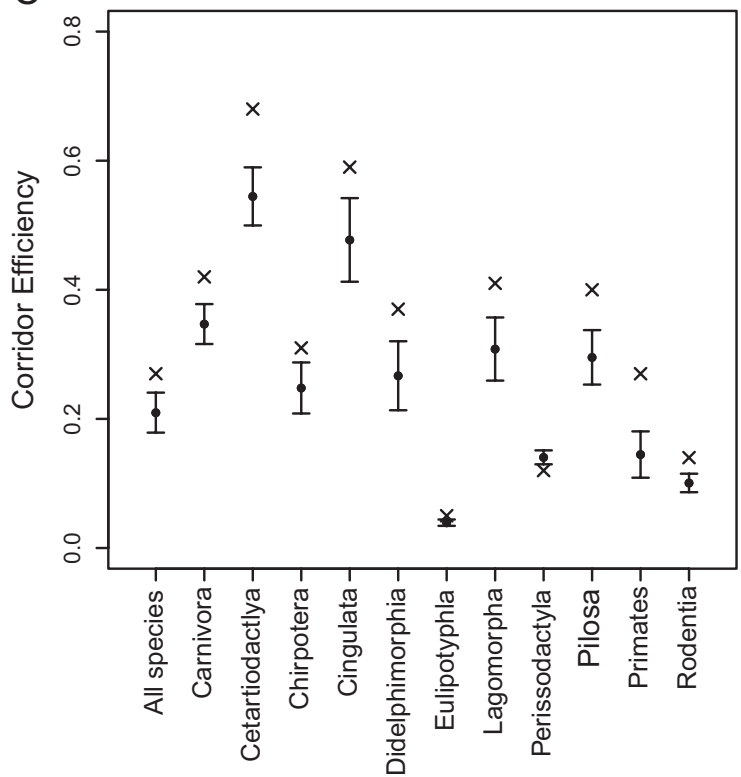

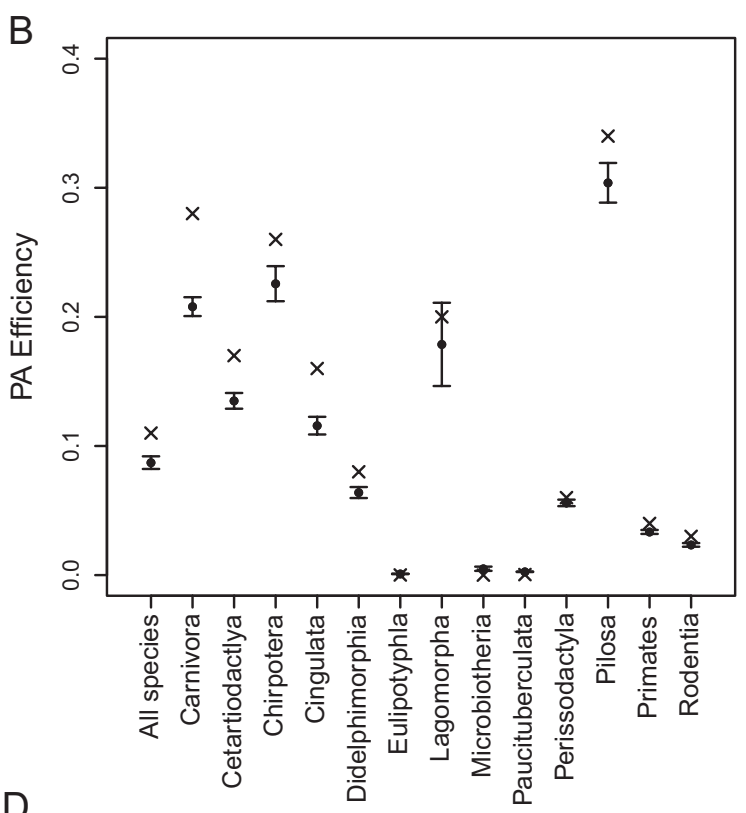

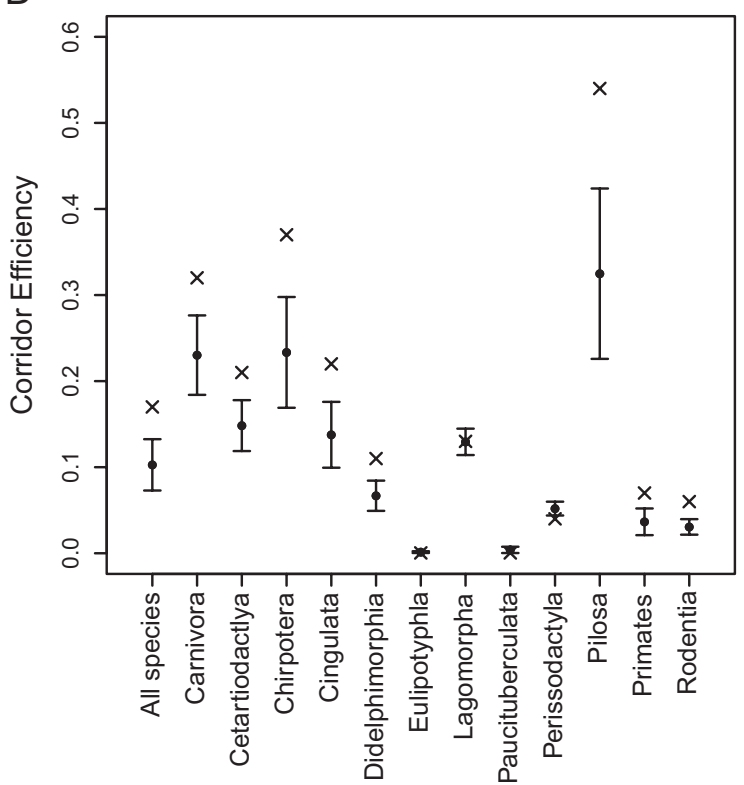

FIG. 4. Comparison of the efficiency of the jaguar network (JCUs and jaguar corridors) and random networks in containing high-quality habitat of co-occurring mammals. Protected area (PA) efficiency and corridor efficiency were calculated as the amount of high-quality habitat for each mammal contained within the network (protected areas or corridors), divided by the total area of the network. Thus, higher values indicate more efficient networks in overlapping high-quality habitat for co-occurring species. Mean and $95 \%$ CI of 10 random networks indicated by solid circles and whiskers; mean value of actual JCU or jaguar corridor network given by the "x". Panel (A) shows a comparison of JCUs in Mexico/Central America with 10 randomly generated protected area networks, (B) a comparison of JCUs in South America with 10 randomly generated protected area networks, (C) a comparison of jaguar corridors in Mexico/Central America with 10 randomly generated corridors, and (D) a comparison of jaguar corridors in South America with 10 randomly generated corridors. Results from an analysis of low- and medium-quality habitat can be found in Appendix S2: Fig. S1.

\section{Conclusions}

A conservation strategy designed around the needs of a single species, the jaguar, can serve as an effective umbrella for conserving high-quality habitat of co-occurring mammal species in Latin America. To our knowledge, this analysis represents one of the first quantitative evaluations of the umbrella value of a single-species, multinational conservation plan and is one of the largest in terms of spatial extent that has been conducted to date. Our ability to analyze data for all co-occurring mammals across the entire range of the jaguar strengthens confidence in our 

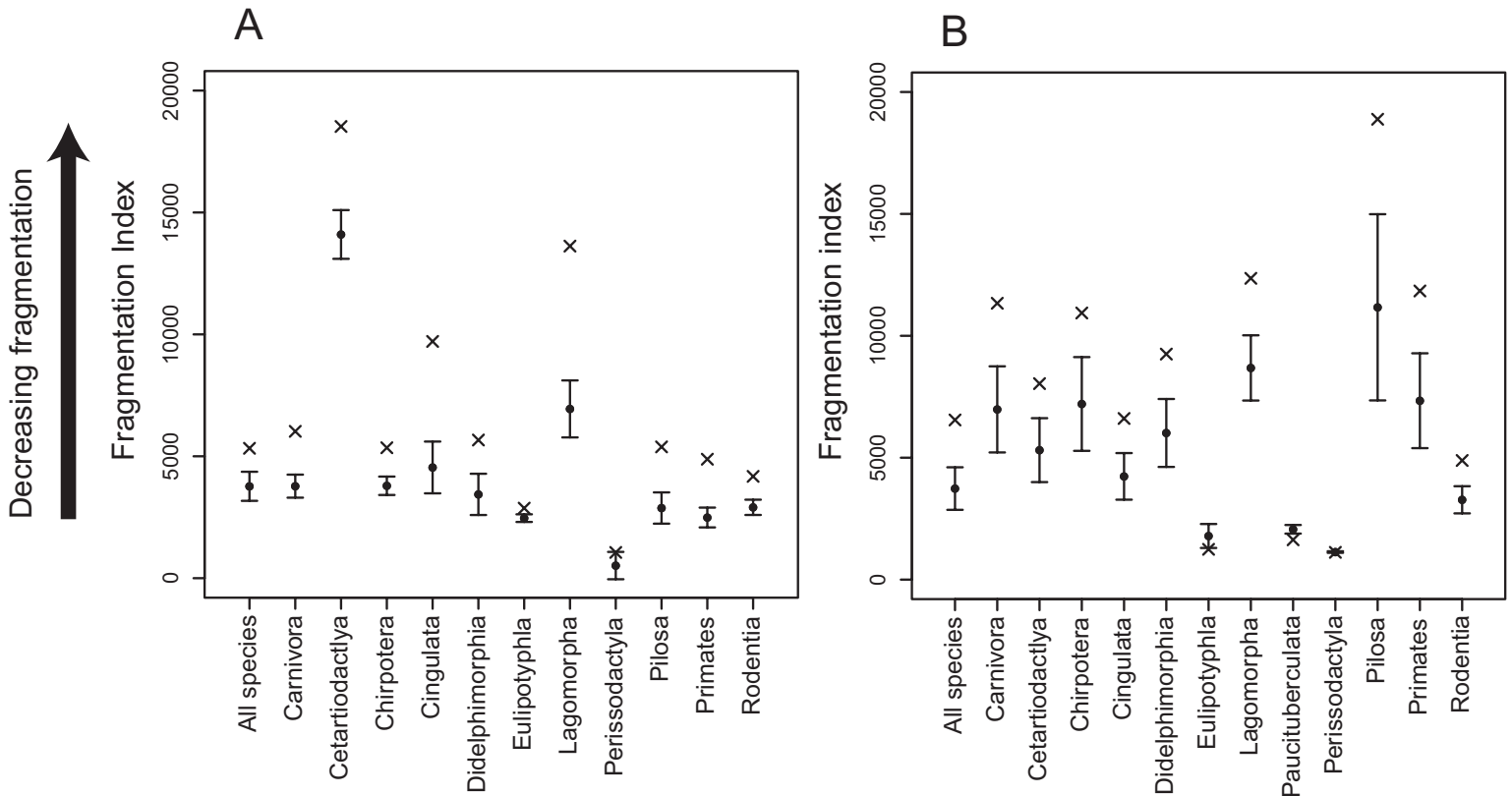

FIG. 5. Comparison of performance of jaguar corridor and random corridors in containing interior, core habitat for co-occurring species. Fragmentation index indicates average distance within high-quality habitat patches to patch edge, thus, greater values indicate less fragmentation. Mean and 95\% CI of 10 random networks indicated by solid circles and whiskers; mean value of actual jaguar corridor network given by the " $x$ ". Panel (A) shows a comparison of jaguar corridors in Mexico/Central America with 10 randomly generated corridors, and (B) a comparison of jaguar corridors in South America with 10 randomly generated corridors.

conclusions. Moreover, because the models developed by Rondinini et al. (2011) measure area of occupancy and habitat quality rather than extent of occurrence, we were able to quantitatively assess umbrella effectiveness at a finer level than simple range limits.

Our results show that the jaguar network outperformed random networks in protecting high-quality habitat for co-occurring mammals. JCUs also conserved a substantial amount and percentage of high-quality habitat; indeed, the percentage of available high-quality habitat of co-occurring mammals that was contained within the JCUs alone was greater than $10 \%$ for virtually all mammalian orders (sometimes substantially so), which is an oft-cited, yet controversial, target threshold for adequate representation of conservation features within a network (Pressey et al. 2003, Brooks et al. 2004, Rondinini et al. 2005). Given the positive (but not necessarily monotonic; Torres et al. 2012) relationship between habitat quality and population density and persistence across a range of scales (Pöyry et al. 2009, Schooley and Branch 2011, Oliver et al. 2012, Robles and Ciudad 2012), and the substantial areal extent of the JCUs (average JCU size $\sim 25000 \mathrm{~km}^{2}$ ), the jaguar network may conserve large populations of many co-occurring mammals. Large population size increases the chance that these populations of co-existing mammals will be viable in the long-term, which is one of the major goals of the umbrella strategy (Caro 2010). In addition, jaguar corridors, due to their substantial width and length, contained additional high-quality habitat, including relatively intact core patches, for mammals outside of JCUs. This pattern suggests that jaguar corridors should facilitate movement for numerous species across the fragmented Latin American landscape. Connectivity is important to ameliorate the effects of habitat loss and fragmentation on co-occurring mammals, and to provide adaptive potential and range shifting in the face of climate change (Groves et al. 2002, Crooks and Sanjayan 2006, Heller and Zavaleta 2009).

Our findings contrast with several studies that have found umbrella species an ineffective conservation strategy (Andelman and Fagan 2000, Roberge and Angelstam 2004, Bifolchi and Lodé 2005, Gardner et al. 2007), including when using wide-ranging species such as large carnivores as umbrellas (Andelman and Fagan 2000, Carroll et al. 2003, Beier et al. 2009, Minor and Lookingbill 2010). We suggest several reasons for this discrepancy. First, and perhaps most importantly, our use of multiple measures of effectiveness was necessary to develop a better understanding of the value of jaguars as an umbrella species. Had we merely investigated species richness as our measure of umbrella effectiveness, using extent of occurrence range maps for mammals as is typically done, we would have concluded that jaguars were not particularly effective umbrellas (except for jaguar corridors in Central America). Random networks performed as well or better at overlapping the ranges of co-occurring mammals. Only upon examination of 
habitat quality of co-occurring mammals did we see the potential value of jaguars as umbrellas. Multiple measures, and metrics that relate more strongly to population viability, may thus be necessary to evaluate the effectiveness of umbrellas in protecting the larger community (Branton and Richardson 2014). Second, the large-scale nature of our assessment likely increased our power of inference. The typical study design for evaluating umbrella effectiveness is relatively small scale, where one or a few reserves occupied by an umbrella are compared with one or a few reserves that are unoccupied. Such studies have revealed mixed results in terms of support for the effectiveness of umbrellas (Branton and Richardson 2010). We suggest that the efficacy of umbrellas should be most evident at larger scales, when guiding the design of the entirety of large conservation networks that will naturally accommodate the needs of many species at regional scales and above. Indeed, several other larger scale studies of umbrella effectiveness revealed support for umbrellas in terms of the number of co-occurring species and amount of those species' range that was contained within an overall conservation network (Noss et al. 1996, Carroll et al. 2001, Rondinini and Boitani 2006, Thorne et al. 2006, but see Andelman and Fagan 2000). Third, we focused our analysis solely on co-occurring mammals, which were taxonomically similar to our umbrella species (also a mammal). Taxonomic relatedness may positively influence conclusions regarding umbrella effectiveness, but this idea is debatable (Fleishman et al. 2001, Hurme et al. 2008, Branton and Richardson 2010). Related to the previous point, a common positive response to tree cover for jaguars and numerous Latin American mammals may be contributing to our results. However, the jaguar network was developed based on consideration of numerous layers in addition to tree cover, including elevation and human disturbance (e.g., hunting pressure, distance to roads and settlements), and therefore, this possibility does not appear to be a sufficient explanation. We also emphasize that while a common response to tree cover may be important, it does not lessen the value of using the jaguar to define the necessary size of protected areas and corridors.

Despite the jaguar network serving as an effective umbrella for mammals in Latin America, the efficacy of the network varies substantially across mammalian orders. Inter-order variability in jaguar umbrella effectiveness was evident for both Mexico/Central America and for South American mammals, although more pronounced for Mexico/Central America. The jaguar network performed better as an umbrella for larger mammals than smaller ones, perhaps due to a better match in scale and type of habitat selection between species of similar size (Wiens 1989). Not surprisingly, the jaguar network conserves a substantial amount of highquality habitat for carnivores and is more effective in conserving high-quality habitat than random networks. The jaguar network also is efficient in conserving high-quality habitat for other mammalian orders, including Cetartiodactyla (even-toed ungulates), Chiroptera (bats), Cingulata (armadillos), Pilosa (anteaters and sloths), and Primates. However, the jaguar serves as a less effective umbrella for mammalian orders such as Rodentia, Lagomorpha (hares and rabbits), and Eulipotyphla (moles and shrews). Numerous species in these last three orders are endangered or threatened due to small range size $(\sim 17 \%$ of species listed as vulnerable, endangered, or critically endangered by IUCN). This finding stresses the need for complementary conservation strategies for these groups. Moreover, although randomly selected networks performed as good as or better than the jaguar network for these mammalian orders, levels of high-quality habitat contained within these random networks were still relatively low, suggesting that additional selection of conservation areas for these particular species-rich mammalian orders is urgently needed.

Inter-order variability in the effectiveness of jaguar corridors in conserving interior, high-quality habitat for co-occurring mammals was also pronounced; however, with the exception of Eulipotyphla, Paucituberculata, and Perissodactyla, jaguar corridors performed better than random corridors. This finding is consistent with two other studies in tropical zones that suggest that large mammals can effectively predict connectivity for other mammalian orders (Epps et al. 2011, Brodie et al. 2014). Our findings, however, contrast with studies in temperate zones that have found limited ability of carnivores to predict connectivity for other mammalian orders (Beier et al. 2009, Minor and Lookingbill 2010, Cushman and Landguth 2012), perhaps because temperate carnivores tend to be highly generalist. Although jaguars are relatively generalized in habitats and diet, they do display a preference for forest cover and aversion to human disturbance, which may increase their ability to predict connectivity for other mammals. In addition, our focus on habitat suitability and fragmentation for sympatric mammals, rather than strict overlap between high connectivity areas or modeled corridors (sensu Beier et al. 2009 and Minor and Lookingbill 2010), or our use of the full co-occurring mammal community in our analysis, may have contributed to our more positive result.

Given our use of large-scale models to assess the effectiveness of jaguars as umbrellas, our results must be approached with a modicum of caution. Our use of random models to compare with the jaguar network may be imperfect but serves as an important starting point for understanding umbrella value of jaguars in comparison to other possible networks. Another approach would be to actually design reserve networks (e.g., Rondinini and Boitani 2006) and corridors to meet the needs of co-occurring mammals and determine the degree of match between the jaguar network and these other networks. However, in addition to the difficulties of such an analysis, particularly for identifying least-cost corridors of other species in a rigorous manner on very 
limited data, the jaguar network was built on expert opinion and includes substantial area outside the world protected area system, making comparisons between networks problematic. We also must be cautious in terms of the relevance of our modeling results to real patterns on the ground. In particular, areas of high-quality habitat based on the Rondinini et al. (2011) models may be impacted by factors not considered in the modeling process (e.g., hunting) that would alter overall quality for numerous mammals (Torres et al. 2012). Also, the jaguar network is a proposed network that is not yet fully realized, and thus, our analysis should be seen as evidence of the potential umbrella value of this modeled network. However, the jaguar network is being actively implemented and refined with field-based ground-truthing and is thus more than a hypothetical model of a reserve network (e.g., Zeller et al. 2010, Petracca et al. 2013, Rabinowitz 2014). This suggests that field-based analysis of the effectiveness of jaguars as umbrellas would be a useful complement to our analysis and should be possible with current data. For example, a substantial amount of camera-trapping data exists that could evaluate species richness or occupancy of co-existing mammals in areas with or without jaguars, or areas of low vs. high jaguar density (sensu Brodie and Giordano 2013). Jaguar camera-trapping has occurred across the jaguar range (Tobler and Powell 2013), and thus, a metaanalytical approach to the umbrella question should result in a larger scale analysis than typical field-based evaluations of umbrella species.

Recent calls for an ecosystem-based approach to conservation planning have suggested that single-species conservation efforts are ineffective (Simberloff 1998, Mac Nally et al. 2002, Hughes et al. 2005). Lack of convincing data regarding the efficacy of surrogate species has contributed to these calls (Mac Nally et al. 2002). Others have suggested that single-species and ecosystembased (or habitat-based) efforts can provide complementary biodiversity conservation strategies (Lindenmayer et al. 2007, Caro 2010). Our findings support this latter conclusion, as we document that the jaguar conservation network can provide substantial, but not complete, umbrella coverage for the co-existing mammal community. This suggests that other range-wide planning exercises for large carnivores may have benefits well beyond protection of a single species. We stress that we do not address the question of whether jaguars are the ideal umbrella species for neotropical or Latin American mammal communities; such an analysis would require that we compare the jaguar network to range-wide conservation strategies developed for several additional threatened species (e.g., white-lipped peccaries, Tayassu pecari), which to date do not exist. While the ideal umbrella species would be an interesting question to address, it might also be a moot point. The jaguar's ability to attract conservation funding and tourism and garner local support will ensure that it is a focus of conservation efforts. Our research indicates that this focus, while somewhat limited, can have far-reaching positive benefits for the entire Latin American mammal community.

\section{ACKNOWLEDGMENTS}

We thank the Murray Lab at Trent University for comments on initial thoughts regarding the umbrella analysis.

\section{Literature Cited}

Adriaensen, F., J. P. Chardon, G. De Blust, E. Swinnen, S. Villalba, H. Gulinck, and E. Matthysen. 2003. The application of 'least-cost' modeling as a functional landscape model. Landscape and Urban Planning 64:233-247.

Andelman, S. J., and W. F. Fagan. 2000. Umbrellas and flagships: efficient conservation surrogates or expensive mistakes? Proceedings of the National Academy of Sciences 97:5954-5959.

Beier, P., D. Majka, and W. D. Spencer. 2008. Forks in the road: choices in procedures for designing wildland linkages. Conservation Biology 22:836-851.

Beier, P., D. Majka, and S. Newell. 2009. Uncertainty analysis of least-cost modeling for designing wildlife linkages. Ecological Applications 19:2067-2077.

Bifolchi, A., and T. Lodé. 2005. Efficiency of conservation shortcuts: an investigation with otters as umbrella species. Biological Conservation 126:523-527.

Branton, M., and J. S. Richardson. 2010. Assessing the value of the umbrella-species concept for conservation planning with meta-analysis. Conservation Biology 25:9-20.

Branton, M. A., and J. S. Richardson. 2014. A test of the umbrella species approach in restored floodplain ponds. Journal of Applied Ecology 51:776-785.

Brodie, J. F., and A. Giordano. 2013. Lack of trophic release with large mammal predators and prey in Borneo. Biological Conservation 163:58-67.

Brodie, J. F., A. J. Giordano, B. Dickson, M. Hebblewhite, H. Bernard, J. Mohd-Azlan, J. Anderson, and L. Ambu. 2015. Evaluating multispecies landscape connectivity in a threatened tropical mammal community. Conservation Biology 29:122-132.

Brooks, T., G. A. B. Da Fonseca, and A. S. L. Rodrigues. 2004. Species, data, and conservation planning. Conservation Biology 18:1682-1688.

Caro, T. 2010. Conservation by proxy: indicator, umbrella, keystone, flagship, and other surrogate species. Island Press, Washington, D.C., USA.

Caro, T. M., and G. O'Doherty. 1999. On the use of surrogate species in conservation biology. Conservation Biology 13:805-814.

Carroll, C., R. F. Noss, and P. C. Paquet. 2001. Carnivores as focal species for conservation planning in the Rocky Mountain region. Ecological Applications 11:961-980.

Carroll, C., R. F. Noss, P. C. Paquet, and N. H. Schumaker. 2003. Use of population viability analysis and reserve selection algorithms in regional conservation plans. Ecological Applications 13:1773-1789.

Che-Castaldo, J. P., and M. C. Neel. 2012. Testing surrogacy assumptions: Can threatened and endangered plants be grouped by biological similarity and abundances? PLoS ONE 7:e1659.

Crooks, K.R. and M. Sanjayan, editors. 2006. Connectivity conservation: maintaining connections for nature. Pages 1-20.Connectivity conservation. Cambridge University Press, New York.

Crooks, K. R., C. L. Burdett, D. M. Theobald, C. Rondinini, and L. Boitani. 2011. Global patterns of fragmentation and 
connectivity of mammalian carnivore habitat. Philosophical Transactions of the Royal Society B 366:2642-2651.

Cushman, S. A., and E. L. Landguth. 2012. Multi-taxa population connectivity in the Northern Rocky Mountains. Ecological Modelling 231:101-112.

De Angelo, C., A. Paviolo, T. Wiegand, R. Kanagaraj, and M. S. Di Bitetti. 2013. Understanding species persistence for defining conservation actions: a management landscape for jaguars in the Atlantic Forest. Biological Conservation 159:422-433.

Epps, C. W., B. M. Mutayoba, L. Gwin, and J. S. Brashares. 2011. An empirical evaluation of the African elephant as a focal species for connectivity planning in East Africa. Diversity and Distributions 17:603-612.

Fleishman, E., and D. D. Murphy. 2009. A realistic assessment of the indicator potential of butterflies and other charismatic taxonomic groups. Conservation Biology 23:1109-1116.

Fleishman, E., R. B. Blair, and D. D. Murphy. 2001. Empirical validation of a method for umbrella species selection. Ecological Applications 11:1489-1501.

Gardner, T. A., T. Caro, E. B. Fitzherbert, T. Banda, and P. Lalbhai. 2007. Conservation value of multiple-use areas in east Africa. Conservation Biology 21:1516-1525.

Groves, C. R., D. B. Jensen, L. L. Valutis, K. H. Redford, M. L. Shaffer, J. M. Scott, J. V. Baumgartner, J. V. Higgins, M. W. Beck, and M. G. Anderson. 2002. Planning for biodiversity conservation: putting conservation science into practice. BioScience 52:499-512.

Heller, N. E., and E. S. Zavaleta. 2009. Biodiversity management in the face of climate change: a review of 22 years of recommendations. Biological Conservation 142:14-32.

Hughes, T. P., D. R. Bellwood, C. Folke, R. S. Steneck, and J. Wilson. 2005. New paradigms for supporting the resilience of marine ecosystems. Trends in Ecology \& Evolution 20:380-386.

Hurme, E., M. Monkkonen, L. Sippola, H. Ylinen, and M. Pentinsaari. 2008. Role of the Siberian flying squirrel as an umbrella species for biodiversity in northern boreal forests. Ecological Indicators 8:246-255.

IUCN, 2006. Conservation strategy for the lion in Eastern and Southern Africa. IUCN SSC Cat Specialist Group, Gland, Switzerland and Cambridge, UK.

Lindenmayer, D. B., et al. 2007. The complementarity of singlespecies and ecosystem-oriented research in conservation research. Oikos 116:1220-1226.

Mac Nally, R., A. F. Bennett, G. W. Brown, L. F. Lumsden, A. Yen, S. Hinkley, P. Lillywhite, and D. Ward. 2002. How well do ecosystem-based planning units represent different components of biodiversity? Ecological Applications 12:900-912.

Minor, E. S., and T. R. Lookingbill. 2010. A multiscale network analysis of protected-area connectivity for mammals in the United States. Conservation Biology 24:1549-1558.

Noss, R. F., H. B. Quigley, M. G. Hornocker, T. Merrill, and P. C. Paquet. 1996. Conservation biology and carnivore conservation in the Rocky Mountains. Conservation Biology 10:949-963.

Oliver, T. H., S. Gillings, M. Girardello, G. Rapacciuolo, T. M. Brereton, G. M. Siriwardena, D. B. Roy, R. Pywell, and R. J. Fuller. 2012. Population density but not stability can be predicted from species distribution models. Journal of Applied Ecology 49:581-590.

Petracca, L. S., E. Ramirez-Bravo, and L. Hernandez-Santin. 2013. Occupancy estimation of jaguar Panthera onca to assess the value of east-central Mexico as a jaguar corridor. Oryx 48:133-140.

Pöyry, J., J. Paukkunen, J. Heliola, and M. Kuussaari. 2009. Relative contribution of local and regional factors to species richness and total density of butterflies and moths in seminatural grasslands. Oecologia 160:577-587.

Pressey, R. L., R. M. Cowling, and M. Rouget. 2003. Formulating conservation targets for biodiversity pattern and process in the Cape Floristic Region, South Africa. Biological Conservation 112:99-127.

Rabinowitz, A. 2014. An indomitable beast: the remarkable journey of the jaguar. Island Press, Washington, D.C., USA.

Rabinowitz, A., and K. A. Zeller. 2010. A range-wide model of landscape connectivity and conservation for the jaguar, Panthera onca. Biological Conservation 143:939-945.

Roberge, J.-M., and P. Angelstam. 2004. Usefulness of the umbrella species concept as a conservation tool. Conservation Biology 18:76-85.

Robles, H., and C. Ciudad. 2012. Influence of habitat quality, population size, patch size, and connectivity on patchoccupancy dynamics of the Middle Spotted Woodpecker. Conservation Biology 26:284-293.

Rondinini, C., and L. Boitani. 2006. Differences in umbrella effects of African amphibians and mammals based on two estimators of the area of occupancy. Conservation Biology 20:170-179.

Rondinini, C., S. Stuart, and L. Boitani. 2005. Habitat suitability models and the shortfall in conservation planning for African vertebrates. Conservation Biology 19:1488-1497.

Rondinini, C., et al. 2011. Global habitat suitability models of terrestrial mammals. Philosophical Transactions of the Royal Society B 366:2633-2641.

Saetersdal, M., and I. Gjerde. 2011. Prioritising conservation areas using species surrogate measures: consistent with ecological theory? Journal of Applied Ecology 48:1236-1240.

Sanderson, E. W., K. H. Redford, C.-L. B. Chetkiewicz, R. A. Medellin, A. Rabinowitz, J. G. Robinson, and A. B. Taber. 2002. Planning to save a species: the jaguar as a model. Conservation Biology 16:58-72.

Sattler, T., G. B. Pezzatti, M. P. Nobis, M. K. Obrist, T. Roth, and M. Moretti. 2013. Selection of multiple umbrella species for functional and taxonomic diversity to represent urban biodiversity. Conservation Biology 28:414-426.

Schooley, R. L., and L. C. Branch. 2011. Habitat quality of source patches and connectivity in fragmented landscapes. Biodiversity and Conservation 20:1611-1623

Sergio, F., I. Newton, L. Marchesi, and P. Pedrini. 2006. Ecologically justified charisma: preservation of top predators delivers biodiversity conservation. Journal of Applied Ecology 43:1049-1055.

Sergio, F., T. Caro, D. Brown, B. Clucas, J. Hunter, J. Ketchum, K. McHugh, and F. Hiraldo. 2008. Top predators as conservation tools: ecological rationale, assumptions, and efficacy. Annual Review of Ecology Evolution and Systematics 39:1-19.

Simberloff, D. 1998. Flagships, umbrellas, and keystones: Is single-species management passé in the landscape era? Biological Conservation 83:247-257.

Thorne, J. H., D. Cameron, and J. F. Quinn. 2006. A conservation design for the central coast of California and the evaluation of mountain lion as an umbrella species. Natural Areas Journal 26:137-148.

Tobler, M. W., and G. V. N. Powell. 2013. Estimating jaguar densities with camera traps: problems with current designs and recommendations for future studies. Biological Conservation 159:109-118.

Torres, N. M., J. De Marco, T. Santos, L. Silveira, A. T. Jacomo, and J. A. F. Diniz-Filso. 2012. Can species distribution modeling provide estimates of population densities? A case study with jaguars in the Neotropics. Diversity and Distributions 18:615-627. 
Wiens, J. A. 1989. Spatial scaling in ecology. Functional Ecology 3:385-397.

Zeller, K. A. 2007. Jaguars in the new millennium data set update: the state of the jaguar in 2006. Wildlife Conservation Society Report, Bronx, NY.
Zeller, K. A., S. Nijhawan, R. Salom-Perez, S. H. Potosme, and J. E. Hines. 2010. Integrating occupancy modeling and interview data for corridor identification: a case study for jaguars in Nicaragua. Biological Conservation 144:892-901

\section{SUPPORTING INFORMATION}

Additional supporting information may be found in the online version of this article at http://onlinelibrary.wiley.com/ doi/10.1890/15-0602.1/suppinfo

\section{Data Availability}

Data associated with this paper have been deposited in Dryad: http://dx.doi.org/10.5061/dryad.8507n 